\title{
PAPER
}

\section{Pallidal deep brain stimulation in patients with cervical dystonia and severe cervical dyskinesias with cervical myelopathy}

\author{
J K Krauss, T J Loher, T Pohle, S Weber, E Taub, C B Bärlocher, J-M Burgunder
}

J Neurol Neurosurg Psychiatry 2002;72:249-256

See end of article for authors' affiliations

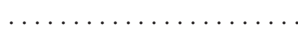

Correspondence to: Professor J K Krauss, Department of Neurosurgery, University Hospital, Klinikum Mannheim,

Theodor-Kutzer-Ufer 1-3, 68167 Mannheim

Germany; joachim.krauss@ nch.ma.uni-heidelberg.de

Received 8 June 2001

In revised form 7

September 2001

Accepted 17 September 2001
Objectives: Surgical treatment of complex cervical dystonia and of cervical dyskinesias associated with cervical myelopathy is challenging. In this prospective study, the long term effect of chronic pallidal stimulation in cervical dystonia and on combining the technique with spinal surgery in patients with severe cervical dyskinesias and secondary cervical myelopathy is described.

Methods: Eight patients with a history of chronic dystonia who did not achieve adequate benefit from medical treatment or botulinum toxin injection participated in the study. Five patients had complex cervical dystonia with tonic postures and phasic movements. Three patients had rapidly progressive cervical myelopathy secondary to severe cervical dyskinesias and dystonia in the context of a generalised movement disorder. Quadripolar electrodes were implanted in the posteroventral lateral globus pallidus internus with stereotactic CT and microelectrode guidance. In the three patients with secondary cervical myelopathy, spinal surgery was performed within a few weeks and included multilevel laminectomies and a four level cervical corporectomy with spinal stabilisation.

Results: Improvement of the movement disorder was noted early after pallidal surgery, but the full benefit could be appreciated only with a delay of several months during chronic stimulation. Three months after surgery, patients with cervical dystonia had improved by $38 \%$ in the severity score, by $54 \%$ in the disability score, and by $38 \%$ in the pain score of a modified version of the Toronto western spasmodic torticollis rating scale. At a mean follow up of 20 months, the severity score had improved by $63 \%$, the disability score by $69 \%$, and the pain score by $50 \%$ compared with preoperatively. There was also sustained amelioration of cervical dyskinesias in the three patients who underwent spinal surgery. Lead fractures occurred in two patients. The mean amplitude needed for chronic deep brain stimulation was $3.8 \mathrm{~V}$ at a mean pulse width of $210 \mu \mathrm{s}$, which is higher than that used for pallidal stimulation in Parkinson's disease.

Conclusions: Chronic pallidal stimualtion is effective for complex cervical dystonia and it is a useful adjunct in patients with cervical dyskinesias and secondary cervical myelopathy who undergo spinal surgery.
$\mathrm{T}$ he renaissance of functional stereotactic surgery for the treatment of Parkinson's disease has also sparked interest in the surgical treatment of other movement disorders. In particular, the surgical treatment of dystonia is currently receiving renewed attention. ${ }^{12}$ Pallidal surgery for dystonia has been reintroduced, and several studies with promising results have been published..$^{3-7}$ More recently, deep brain stimulation (DBS) through chronically implanted electrodes in the globus pallidus internus has gained increasing importance in the treatment of patients with medically intractable dystonia. ${ }^{8-11}$ So far, however, most studies have reported only on relatively short follow up periods and are uncontrolled.

The treatment of chronic cervical dystonia (CD) can be difficult. Medical treatment often provides limited benefit and may be burdened by intolerable side effects. Nowadays, the treatment of choice is the local injection of botulinum toxin type A into the dystonic muscles. ${ }^{12}{ }^{13}$ However, this therapeutic approach is ineffective in certain patients or loses its efficacy with repeated injections. ${ }^{14}$ Contemporary surgical treatment includes several procedures such as intradural anterior rhizotomy, extradural ramisectomy, and selective denervation, as well as myectomy and myotomy. ${ }^{15-21}$ Serial selective procedures tailored to the individual pattern of dystonic activity can achieve varying long term benefit with low permanent morbidity. ${ }^{22}$ Peripheral surgery, however, is not suited in a subset of patients with complex CD. ${ }^{15}$ Also, it is not considered useful in patients who present with marked cervical dyskine- sias in the context of generalised choreoathetotic movement disorders. These patients may develop degenerative spine changes with secondary neurological deficits-that is, cervical myelopathy and radiculopathies. ${ }^{23}{ }^{24}$ Spinal surgery under such circumstances is problematic, in particular when the underlying movement disorder cannot be treated appropriately. Review of the history of functional stereotactic surgery for $\mathrm{CD}_{1}{ }^{25}$ and our experience with pallidal surgery in patients with Parkinson's disease with dystonic dyskinesias and in patients with generalised dystonia, prompted us to investigate its potential in complex CD.

The purpose of this prospective study was to evaluate the effect of chronic bilateral DBS of the globus pallidus internus in patients with complex CD. Here, we discuss the rationale for pallidal DBS in CD, describe the operative techniques, and report on a series of patients with clinical follow up up to 30 months. Preliminary data on the first few patients, with limited follow up, have been published elsewhere. ${ }^{26}$ We also describe the usefulness of the technique in patients with severe cervical dyskinesias undergoing spinal surgery for the treatment of secondary cervical myelopathy.

Abbreviations: $D B S$, deep brain stimulation; $C D$, cervical dystonia; TWSTRS, Toronto western spasmodic torticollis rating scale; MMSE, mini mental state examination; CDRS, cervical dyskinesia rating scale 
Table 1 Clinical characteristics of patients with idiopathic cervical dystonia selected for bilateral pallidal deep brain stimulation

\begin{tabular}{|c|c|c|c|c|c|}
\hline $\begin{array}{l}\text { Patient } \\
\text { No }\end{array}$ & Sex & Age & $\begin{array}{l}\text { Duration } \\
\text { of } C D(y)\end{array}$ & Pattern of cervical dystonia & Response to botulinum toxin injections \\
\hline 1 & 53 & $\mathrm{~F}$ & 6 & Retrocollis + torticollis & Primary non-responder \\
\hline 2 & 47 & M & 4 & Sagittal shift + retrocollis & $\begin{array}{l}\text { Initially mild response, difficulty with injection of deep muscles, } \\
\text { secondary non-responder }\end{array}$ \\
\hline 3 & 41 & $\mathrm{~F}$ & 5 & Lateral shift + lateral tilt & Moderate response, patient not satisfied with result \\
\hline 4 & 28 & M & 6 & Antecollis & $\begin{array}{l}\text { Mild response, difficulty with injection of deep muscles, patient not } \\
\text { satisfied with result }\end{array}$ \\
\hline 5 & 46 & M & 24 & Bilateral phasic oscillating torticollis & Primary non-responder \\
\hline
\end{tabular}

\section{PATIENTS AND METHODS \\ Patients}

All patients with CD enrolled in this study were regularly seen in our movement disorders clinic before surgery. Only those patients who were thought not to be good candidates for peripheral surgical techniques were considered candidates for pallidal DBS. The study protocol was approved by our institutional human investigation committee, and all patients gave their written informed consent to participation in the study.

The clinical characteristics of the five patients with cervical dystonia are detailed in table 1. Mean age at surgery was 43 years. The mean duration of dystonia up to the time of surgery was 9 years (range 4-24 years). All patients had complex manifestations of CD with tonic postures and phasic head movements. Phasic movements were only mild in one patient, but moderate or severe in three patients. Patient 5 had a very unusual type of CD involving bilateral phasic oscillating torticollis. All patients had unremarkable MRI of the brain and were considered to have idiopathic dystonia. Patient 5 had two brothers affected with dystonia; all other patients had a negative family history. Subtle dystonic movements of other body parts were seen in three patients (the shoulder in one patient and the face in two patients). At the time of surgery, none of the patients had a useful response to botulinum toxin injections, for reasons listed in table 1. All patients had been treated with anticholinergic drugs, with either an inadequate response or intolerable side effects. Other medications used to treat dystonia in this patient group included diazepam, prazepam, bromazepam, lorazepam, chlordiazepoxide, tetrabamate, flupentixol, melitracen, baclofen, chlorzoxacon, tizanidine, diphenhydramine, propranolol, tetrabenazine, and levodopa.

The three patients with secondary cervical myelopathy due to movement disorders affecting the cervical spine were all disabled. They were scheduled both for spinal surgery and functional stereotactic surgery for treatment of their underlying movement disorder. Two of these patients were women, 46 and 40 years old, with severe cervical dyskinesias due to choreoathetoid infantile cerebral palsy. They had developed neurological deficits over several years and were both wheelchair bound on admission. Magnetic resonance studies of the cervical spine in both patients showed multilevel spondylosis and spinal stenosis with hyperintense intramedullar lesions on T2 weighted sequences. Both patients had a rapid progression of paraparesis over a period of about 6 months before their referral. In one of these patients, myelography showed a complete block at $\mathrm{C}$. The third patient with secondary cervical myelopathy was a 46 year old man with prominent CD in the context of generalised dystonia. He had undergone intradural rhizotomy and right sided thalamotomy in his adolescence resulting in temporary improvement of dystonia. At the age of 38, he developed spinal ataxia; imaging studies showed severe cervical spondylosis, with instability at the C3-4 and C4-5 levels (fig 1 A). He underwent anterior plate stabilisation from $\mathrm{C} 3$ to $\mathrm{C} 5$ and had a satisfactory radiographic result ( fig l B). The movement disorder, however, was not treated surgically at this time. Over the next few years he became severely disabled because of progressive tetraparesis and sensory loss. In the year preceding referral, he became nearly unable to walk and sustained several fractures because of repeated falls. Radiographs showed loosening of the screws and displacement of the plate, with marked kyphotic angulation and subluxation from C2 to C7 (fig l C). Spinal MR images showed narrowing of the spinal canal from C2 to C7, with cord traction across the kyphotic zone.

\section{Study protocol and follow up analysis}

Patients with idiopathic CD were assessed preoperatively and at defined follow up visits according to a standard protocol including a modified Toronto western spasmodic torticollis rating scale (TWSTRS), as used earlier, consisting of subscores for the severity of CD (possible maximal score 32), functional disability (possible maximal score 60), and pain (possible maximal score 8$)^{22} 27$; mini mental state examination (MMSE); Hamilton depression scale; and a standard videotape protocol. The TWSTRS could not be used for the patient with bilateral oscillating phasic torticollis. All formal assessments were performed by the neurologists on the study team.

Patients with cervical dyskinesias and cervical myelopathy were assessed with a cervical dyskinesia rating scale (CDRS) adapted from the Burke-Fahn-Marsden scale. ${ }^{28}$ This scale has not been validated thus far. The severity of dyskinesias at rest and during movement was graded on a scale of 0 to 4 . An overall score was then calculated according to the following formula: ( $\%$ of time with dyskinesia $) \times(4 \times($ severity at rest $))+(\%$ of time with dyskinesia $) \times(3 \times($ severity during movement)). Thus, the maximum possible overall CDRS score was 28.

Clinical assessments were made preoperatively, 1 week, and 3 months after the operation, and at subsequent 3 month intervals up to 1 year postoperatively. Long term follow up assessments were planned at 6 month intervals subsequently. New assessments were also made preoperatively in those patients who needed replacement of implantable pulse generators because of depletion of the batteries. For statistical analysis the paired $t$ test and the Wilcoxon signed rank test were used.

\section{Surgical procedure of DBS of the globus pallidus internus}

The basic principles and rationales of our stereotactic technique have been described in detail previously. ${ }^{29-31}$ All patients underwent simultaneous bilateral surgery except the one patient who had previously undergone thalamotomy. Preoperatively, the morphology of the basal ganglia was assessed with multiaxial $\mathrm{T} 1$ inversion recovery and $\mathrm{T} 2$ weighted MRI. A stereotactic frame was affixed to the patient's head under local anaesthesia. Four patients required temporary sedation with intravenous midazolam for fixation of the frame and stereotactic imaging, because the phasic head movements interfered markedly. The anterior and posterior commissures were identified on contiguous $1 \mathrm{~mm}$ stereotactic 
axial CT scans which were transferred to a work station to allow inspection of coordinated simultaneous, reformatted sagittal and coronal views. Preliminary targets in the left and right lateral posteroventral globus pallidus internus were chosen at $19-21 \mathrm{~mm}$ lateral to the midline, 3-5 mm below the intercommissural line, and $2-3 \mathrm{~mm}$ anterior to the midcommissural point using an algorithm to correct for misalingment of the intercommissural line relative to the frame axes. ${ }^{31}$ Patients were put in the semisitting position with the frame fixed to the operating table, in a position where they did not have to resist dystonic pulling of the head. Precoronal burr holes were made bilaterally $2 \mathrm{~cm}$ from the midline. The arachnoid was opened at the crown of a gyrus and a guiding cannula was inserted stereotactically. Microelectrode recording was performed with high impedance electrodes to identify and map single unit records from $20 \mathrm{~mm}$ above the target to 5 $\mathrm{mm}$ below the target. Accordingly, the borders of the putamen, globus pallidus externus, and globus pallidus internus were defined. The optic tract was identified by microstimulation. Depending on the quality of the recordings and on the distribution of the neural activity, one to three microelectrode trajectories were explored on each side. After definition of the ventral border of the globus pallidus internus, a quadripolar electrode (model 3387, Medtronic Inc, Minneapolis, MN, USA) was inserted through the guiding cannula. The lowest electrode contact was placed at the refined target at the ventral
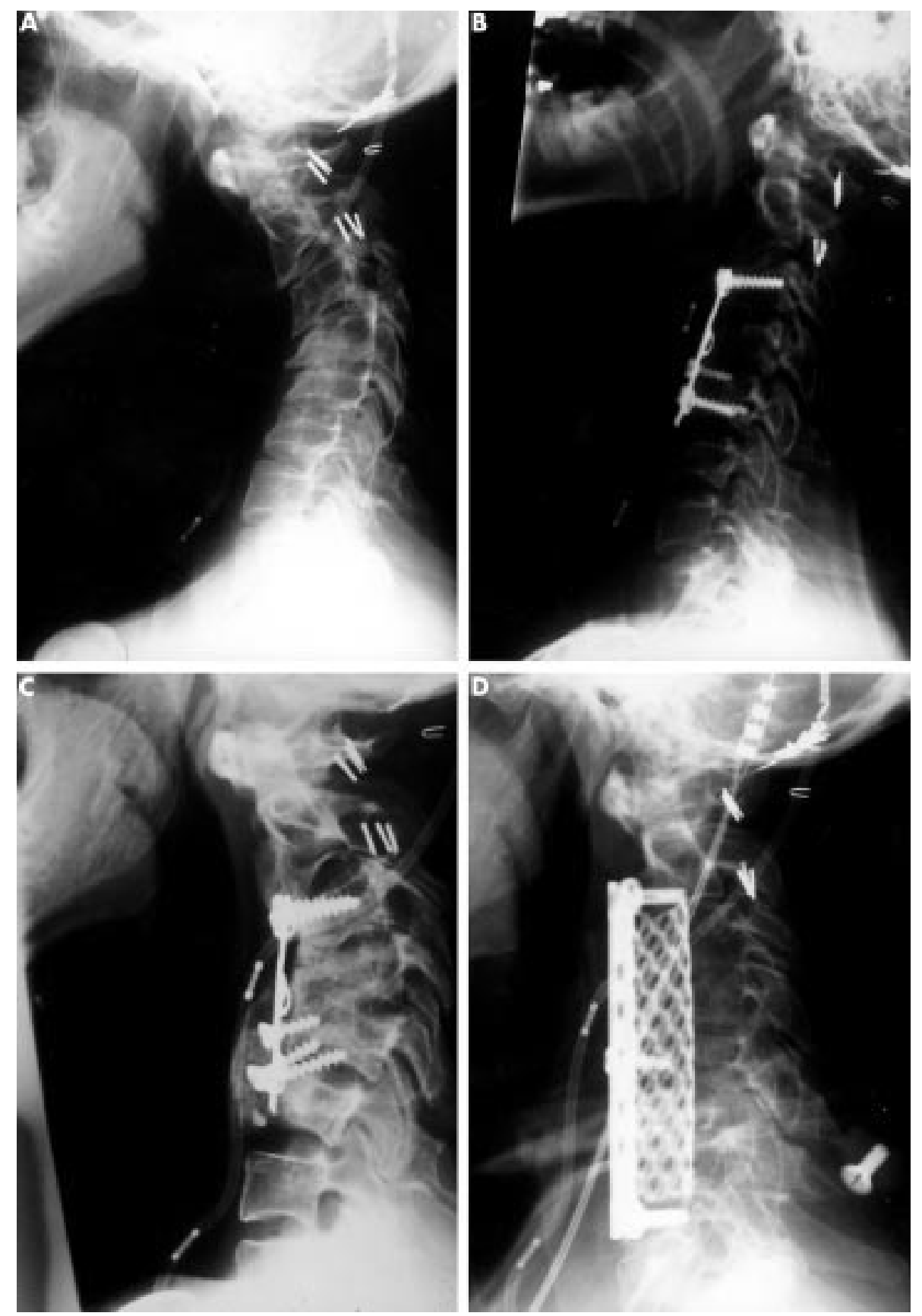

Figure 1 Lateral radiographs of a 46 year old man with cervical myelopathy due to severe cervical dystonia. (A) Kyphotic angulation and subluxation from $\mathrm{C} 3$ to $\mathrm{C} 5$ at the age of 38 . (B) Correction of the deformity by anterior plate stabilisation. (C) Six years later, the plate and the screws are displaced and there is a severe spinal deformity from C2 to C7. (D) Postoperative result after removal of old hardware, four level corporectomy, stabilisation with a titanium cage filled with autologous bone, plating from $\mathrm{C} 2$ to $\mathrm{C} 7$, and postoperative halo immobilisation. The patient underwent pallidal DBS before the spinal procedure. 
pallidal border locating the tip of the lead (dead space) $1.5 \mathrm{~mm}$ below the target. Macrostimulation for extrinsic effects was performed directly with the quadripolar electrode at a pulse width of $210 \mu \mathrm{s}$ at frequencies of $5 \mathrm{~Hz}$ and $100 \mathrm{~Hz}$. The voltage was gradually increased until optic or capsular responses were evoked. Electrode position was corrected in case the thresholds for the occurrence of such extrinsic effects were too low. It was not possible to reliably control the effect of the stimulation on the movement disorder intraoperatively. The electrode was fixed to the skull with the standard plastic burr hole ring and cap and its distal end was coiled under the parietal galea. Then the other side was approached accordingly. The scalp wounds were closed, the stereotactic frame was removed, and general anaesthesia was induced. No interoperative test stimulations were performed. All patients received bilateral implantable pulse generators except the patient who had a previous thalamotomy. The implantable pulse generators (Itrel II, Medtronic) were connected to the corresponding electrodes through subcutaneous tunnelled extension cables and placed in an infraclavicular subcutaneous pouch.

\section{Postoperative care}

All patients had routine postoperative CT to rule out haemorrhage or other adverse events. Contiguous $1 \mathrm{~mm}$ axial scans were obtained through the region of the third ventricle. Transferral of these scans to a work station allowed localisation of the electrode contacts in relation to the intercommissural line.

The implantable pulse generators were programmed bilaterally on the first postoperative day. The initial settings were usually as follows: symmetric bipolar stimulation with contact 1 negative and contact 2 positive, pulse width $210 \mu$ s, frequency $130 \mathrm{~Hz}$, and amplitude $1 \mathrm{~V}$. Over the next 5 days, the amplitudes were gradually increased up to 2-3 V. On subsequent follow up visits, the stimulation settings were adjusted. Overintense stimulation produced undesired effects, usually a feeling of perioral tightness. Amplitudes below the thresholds at which this occurred were programmed. By the time of the patient's next visit, the stimulation settings were readjusted, including also different values for pulse width and frequency.

\section{RESULTS}

\section{Early postoperative course}

There were no intraoperative or postoperative complications. The postoperative CT showed the correct electrode position in all but one patient, in whom an electrode was considered to be too deep on one side and was pulled back $2 \mathrm{~mm}$ in a second procedure under local anaesthesia. In general, some degree of improvement of CD and dyskinesias could be seen as soon as the patients recovered from anaesthesia. There was mild to moderate improvement of the dystonic posture, and more pronounced improvement of phasic movements. This beneficial effect was found even before the stimulation was turned on and thus might have been due to a micropallidotomy effect.

\section{Clinical outcome of patients with cervical dystonia}

All patients were available for regular follow up examinations. The dystonia improved gradually over the next 6 months during adaptation of the stimulation settings. Phasic components improved earlier, and dystonic postures improved later. A representative example is shown in figure 2 . The subtle dystonic movements of body parts other than the neck disappeared after prolonged stimulation. Anticholinergic medication was tapered off in all patients during follow up, and benzodiazepines in two patients were reduced. The first patient in this series had several exacerbations of CD in the late follow up (after 18 months). Two such episodes were due to unilateral electrode fractures, and two to implantable pulse generator battery depletion. Each time, CD had become worse within a
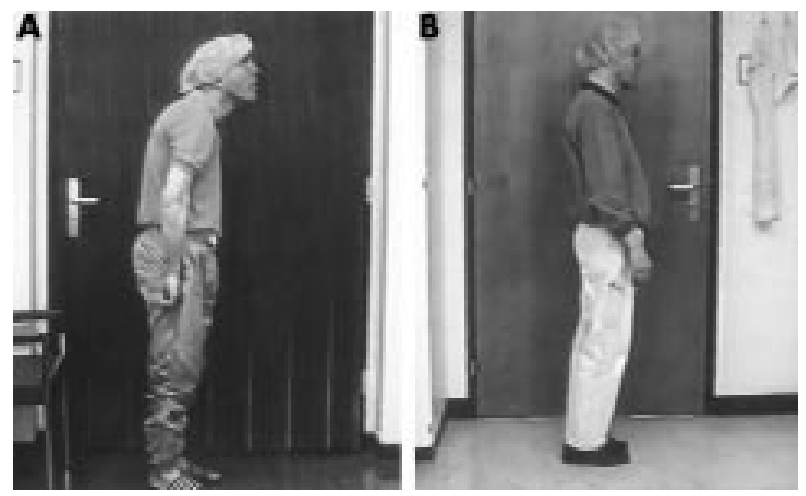

Figure 2 Video stills of a 47 year old man with complex cervical dystonia characterised mainly by sagittal shift and retrocollis, before and 9 months after bilateral pallidal stimulation.

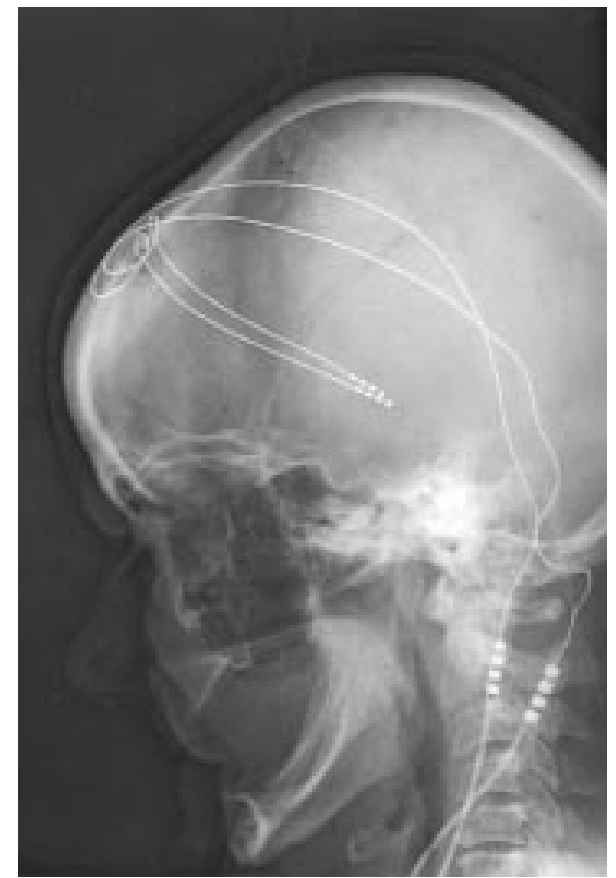

Figure 3 Radiography of patient 1 showing a fracture of the left lead just above the connection to the extension wire. The electrode was replaced stereotactically. The connector was placed behind the ear in subsequent procedures.

few hours. After replacement of the non-functioning hardware, clinical improvement was noted to occur with much less delay than before. Patient 5, with bilateral oscillating phasic torticollis, had a more variable course. The dystonic movements were minimal during the first 3 months after surgery, but later recurred on several occasions and finally improved only after extensive reprogramming of the stimulator. Improvement has been stable in the other three patients.

The gradual and progressive postoperative improvement of CD is reflected by evaluation with the TWSTRS. Mean preoperative and postoperative subscores of the modified TWSRTS are shown in table 2 . The last available TWSTRS assessment was at 12 months postoperatively, at 18 months, at 20 months, and at 30 months. All subscores were improved substantially by 3 months after surgery; the severity score had improved by $38 \%$, the disability score by $54 \%$, and the pain score by $38 \%$. All subscores were improved still further on the last follow up evaluation. At the last follow up, the severity score had improved by $63 \%$, the disability score by $69 \%$, and the pain 
Table 2 Subscores of the modified Toronto western spasmodic torticollis rating scale in patients with idiopathic cervical dystonia before and after bilateral pallidal deep brain stimulation

\begin{tabular}{|c|c|c|c|c|c|c|}
\hline & \multirow[b]{2}{*}{ Max score } & \multirow[b]{2}{*}{ Preoperative } & \multicolumn{4}{|c|}{ Postoperative } \\
\hline & & & 3 months & 6 months & 12 months & Last follow up $†$ \\
\hline Total severity score & 32 & 20.5 & $12.7 * * *$ & $9.7 * * *$ & $9.2 * * *$ & $7.5^{* * *}$ \\
\hline Total functional disability score & 60 & 40.5 & $18.5^{* *}$ & $17.2 * * *$ & $13.7 * * *$ & $12.7 * * *$ \\
\hline Total pain score & 8 & 6 & 3.7 (NS) & 3.5 (NS) & $3.3^{*}$ & $3^{*}$ \\
\hline
\end{tabular}

score by $50 \%$ compared with those preoperatively. The difference in the subscores at the last follow up compared with the 3 month follow up was significant for the severity and the functional disability subscores, but not for the pain subscore. One patient was completely pain free at the last follow up, but all other patients still complained of residual neck pain or tension. As all patients had some, albeit minor or negligible, dystonic posture on follow up there was no significant change in the subitem percentage of time present in the TWSTRS severity subscore. In the functional disability subscore, there were significant improvements for the subitems driving, reading, writing, watching TV, outside activities, drinking, eating, personal hygiene, work/domestic work, and dressing. Two patients returned to work. The mean scores for verbal communication and social embarrassment were not significantly improved.

The mean preoperative MMSE score was 27 . This was partly due to the movement disorders interfering with performance of the test in some cases. The MMSE scores reached the highest possible value at the subsequent follow up examinations (table 3). Preoperatively, all patients except one were mildly depressed as reflected by their Hamilton depression scores. During follow up, there was a continuous improvement of these scores which paralleled the improvement of the dystonia (table 3). The changes of the MMSE and Hamilton depression scores did not reach statistical significance.

\section{Clinical outcome of patients with cervical dyskinesias and cervical myelopathy}

Functional stereotactic surgery was performed 3 months before spinal surgery in two patients, and 1 month after spinal surgery for acute deterioration of the neurological deficits in one patient. The two patients with cervical dyskinesias due to choreoathetoid infantile cerebral palsy underwent multilevel laminectomies, at C3-C5 in one case and at C7-T1 in the other case. The patient with prominent CD in the context of generalised dystonia and failed previous stabilisation underwent an extensive procedure involving removal of old hardware, C3-C6 corporectomy, stabilisation with a titanium cage filled with autologous bone, C2-C7 anterior plating, and halo immobilisation for 6 months (fig 1 D). There were no intraoperative complications or new postoperative deficits. The patient who had acute deterioration of her neurological deficits before laminectomy recovered to her earlier level within 3 months.

In both patients with choreoathetoid infantile cerebral palsy, the severity and frequency of the cervical dyskinesias improved with bilateral DBS of the globus pallidus internus. The improvement at 12 and 18 months was no better than that at 3 months and was less marked than that seen in the patients with idiopathic CD. The mean CDRS scores at rest improved by $50 \%$ at 1 year (mean preoperative score, $8 ; 3$ month follow up, 3.4; 6 month follow up, 3.4; and 12 month follow up, 4). The mean CDRS scores during movement improved by $40 \%$ at 1 year (mean preoperative score, 10; 3 month follow up, 6; 6 month follow up, 6; and 12 month follow up, 6). The patient with prominent CD in the setting of generalised dystonia tolerated halo immobilisation well with unilateral globus pallidus internus DBS. The dystonic posture had almost disappeared by the time the halo was removed. At 24 months after surgery, the improvement is stable and radiographs of the cervical spine show solid fusion without instability.

Progression of cervical myelopathy was halted in all three patients at the last follow up, and symptomatic improvement of the movement disorder was sustained. Although patients subjectively reported better performance in certain activities of daily living, no significant change was found in formal assessment of functional disability. The two patients with infantile cerebral palsy were able to participate again in daily activities in their sheltered home. The patient with extensive spinal reconstruction returned to living at home.

\section{Stimulation programming and DBS related events}

As indicated above, the adjustment of DBS settings was time consuming in some instances. At 3 months postoperatively, the mean number of programming sessions was 4.8/patient (range 4-7), and at 12 months the mean number of visits was 8.8 (7-15). In general, no further programming was performed later than 9 months postoperatively. The mean values of the settings at the last follow up were amplitude $3.8 \mathrm{~V}$ (3.0$4.5)$, frequency $135 \mathrm{pps}$ (130-145), and pulse width $210 \mu \mathrm{s}$. All patients except one had bipolar stimulation. Three lead fractures occurred, two of them in one patient. The fracture was on the quadripolar DBS electrode directly above its connection to the extension cable (fig 3). This connection had been placed in the subcutaneous tissue of the neck in the first three patients. The electrodes were replaced stereotactically under local anaesthesia. The implantable pulse generators had to be replaced because of battery depletion in two patients, at 22 months and at 25 months. At this time the Itrel IIs were replaced by a Kinetra (Medtronic) which allows stimulation through two electrodes with a single pacemaker. Eighteen

Table 3 Mini mental state examination and Hamilton depression scale in patients with idiopathic cervical dystonia before and after bilateral pallidal deep brain stimulation

\begin{tabular}{lllllll}
\hline & \multicolumn{7}{c}{ Postoperative } & & \\
\cline { 5 - 6 } & Max score & Preoperative & 3 months & 6 months & 12 months & Last follow up* \\
\hline Mini mental state examination & 30 & 27 & 29 & 30 & 30 & 30 \\
Hamilton depression scale & 52 & 9 & 4.5 & 5.7 & 5.7 & 3 \\
\hline *Mean of last follow up=20 months. Changes did not reach statistical significance. & & &
\end{tabular}


months after implantation of the DBS sytem, one patient had a subcutaneous infection above the connector, which had been placed behind the ear. Systemic antibiotics were administered for 2 weeks and the infection healed uneventfully.

No patient had a permanent complication due to the surgical procedures or to chronic DBS. Six patients experienced a feeling of perioral tightness at some point during adjustment of the DBS settings. This was sometimes associated with mild dysphagia and difficulty in speaking. It could always be eliminated by decreasing the voltage of the stimulation. Two patients experienced excessive weight gain during chronic pallidal DBS. One of these patienst had lost about 40 pounds preoperatively because he had difficulty swallowing with his dystonic neck posture.

\section{DISCUSSION}

The treatment options to date for patients with complex CD and cervical dyskinesias have been limited. This prospective study shows that bilateral pallidal DBS can produce both symptomatic and functional improvement, including marked relief of pain in this group of patients for up to 2.5 years. The improvement in the patients with idiopathic CD corresponds to the experience obtained in patients with other types of dystonia who underwent pallidal surgery. In particular, patients with DYT-1 positive dystonia experienced excellent improvement after pallidotomy or pallidal DBS. ${ }^{39}$ Patients with primary dystonia benefit in general from pallidal surgery. ${ }^{4} 711$ The lesser improvement of cervical dyskinesias in patients with choreoathetosis is also consistent with previous experience of pallidal surgery for dystonia. It has been suggested that the response of dystonia to pallidal surgery may depend on aetiology; patients with primary dystonia respond well, patients with secondary dystonia without a structural lesion respond less well, and poor results are achieved in patients with secondary dystonia and structural lesions. ${ }^{32}$ Although our patients had immediate relief in the early postoperative period, the full benefit of chronic DBS could only be appreciated over the next few months during adjustment of the DBS settings. This delayed improvement has also been seen in patients with other types of dystonia. Our positive experience with chronic DBS of the globus pallidus internus for complex CD accords with the limited experience reported by others. ${ }^{32-34}$ In one study on two patients a dissociation between pain and motor improvement was found..$^{35}$

In the historical heyday of functional stereotactic surgery, patients with various movement disorders were treated with stereotactic lesions in the thalamus and basal ganglia. ${ }^{25}$ The selection of the site and side to be operated on depended on the current hypotheses and beliefs concerning the pathophysiology of CD. Hassler and Dieckmann developed an elaborate theory on the correlates of specific types of CD in analogy with findings from animal experiments. ${ }^{36}{ }^{37}$ They thought that the target should be selected according to the phenotype of CD in the individual patient. Targets used by different surgeons included the ventral lateral nuclei of the thalamus (ventrooralis anterior, ventro-oralis posterior, and ventro-oralis internus); the centre median nucleus; the ventralis intermedius; the ventralis posteromedialis, and posterolateralis; the pulvinar; Forel's fields $\mathrm{Hl}$ and $\mathrm{H} 2$; the zona incerta; the interstitial nucleus of Cajal; and the red nucleus. The difficulties in choosing the appropriate side and site are exemplified by contradictory recommendations of two historic authorities. Whereas Cooper recommended lesioning contralateral to the dystonic sternocleidomastoid muscle, ${ }^{38}$ Hassler and Dieckmann stated that the ipsilateral side should be targeted. ${ }^{36}{ }^{37}$ Overall, about 300 patients with CD were reported to have undergone functional stereotactic surgery in the 1960s and 1970s. Although the historical reports are difficult to interpret retrospectively, it seems that most patients had mild to moderate benefit. Mundinger reported on his experience with thalamic DBS for CD as early as $1977 .{ }^{39}$ The results, however, never appeared in the English literature and the study went unnoticed. The technique was then abandoned because of hardware difficulties. After the work of Benabid et al and Siegfried and Lippitz, DBS has become an accepted alternative to lesioning procedures. ${ }^{40} 41$

When planning this study, we considered several alternatives regarding the choice of the target and the surgical method. We chose the pallidum rather than the thalamus by analogy with the experience we gained in patients with Parkinson's disease and other types of dystonia. Patients with Parkinson's disease, in our experience, may benefit strikingly from improvement of their off period dystonia after pallidal surgery (unpublished data). According to recent reports, thalamic surgery might also be reconsidered to treat dystonia, and might be a better option than pallidal surgery in secondary dystonia. ${ }^{42}$ We decided to use chronic DBS instead of ablative surgery for several reasons. Firstly, when the study was initiated there was no other current experience with pallidal surgery for treatment of $C D$, and we preferred to use a reversible technique. Secondly, we thought that chronic DBS would be safer for bilateral surgery. Thirdly, DBS variables can be adjusted until an optimal response is achieved and the target can be changed by selection of different electrode contacts.

The pathophysiology of dystonia is poorly understood.$^{43}$ It is generally thought that, similarly to secondary dystonia, idiopathic dystonia is due to dysfunction of the basal ganglia or of the brain stem. ${ }^{44}$ Disturbance of the indirect striatopallidal pathway, resulting in disinhibition of thalamocortical activity, has been proposed. Recent PET investigations have shown higher glucose metabolism bilaterally in the lentiform nucleus in patients with CD without significant differences regarding the laterality, the specific pattern, or the severity of $\mathrm{CD}$ in individual cases. ${ }^{45}$ Bilateral basal ganglia involvement in CD has also been suggested by a recent SPECT study ${ }^{46}$ Striatal D2 receptor binding was significantly reduced in patients with CD compared with normal controls. No significant difference, however, was found by intraindividual comparison of contralateral versus ipsilateral striatal epipride binding with regard to the direction of head rotation.

Bilateral rather than unilateral surgery is also supported by accumulating knowledge on the innervation of neck muscles. Earlier animal studies on ipsilateral versus contralateral control of the sternocleidomastoid muscle yielded inconsistent results. ${ }^{47}$ In a recent transcranial magnetic stimulation study in normal subjects, however, ipsilateral as well as contralateral sternocleidomastoid responses were evoked by stimulation of an area of cortex near the representation of the trunk.$^{48}$ Another study showed that, after amytal injection of the right internal carotid artery, all patients who were fully cooperative were able to lift and turn their heads both to the right and to the left on command..$^{49}$ Ten of the 14 patients had weakness of the right sternocleidomastoid muscle compared with the left, suggesting that most of the innervation of the sternocleidomastoid comes from the ipsilateral hemispere.

The occurrence of secondary neurological deficits in patients with movement disorders affecting the cervical spine is often ignored. ${ }^{17}$ Many of these patients are referred only late when they are already wheelchair bound or when they have developed other severe neurological deficits. Also, they are often not considered candidates for spinal surgery because it is assumed that only minor benefit is achieved and the risk of surgical complications and spinal instability is higher than in other patients. The second assumption is probably justified, because there is continued mechanical stress on the cervical spine when the hyperkinetic movement disorder is not treated as well. Prolonged improvement of the movement disorder would be desirable in this difficult to treat group of patients. Delayed dislodgement of methylmethacrylate resulting in 
additional neurological deficits has been described after spinal surgery for choreoathetotic infantile cerebral palsy, ${ }^{23}$ and, as in one of the patients discussed here, spinal stabilisation without treatment of the underlying movement disorder may not result in long term benefit. It has been debated whether anterior or posterior approaches should be preferred in spinal surgey on patients with choreoathetosis. ${ }^{23}{ }^{24}$ Extensive spinal reconstruction procedures are clearly a treatment of last resort. Chronic pallidal DBS seems to be a useful adjunct in this unfortunate situation. Although the improvement of the involuntary movements in the patients with infantile cerebral palsy was less striking than in idiopathic $C D$, the improvement of the incessant dyskinesias, combined with spinal surgery, allowed social reintegration of these patients.

The efficacy of pallidal DBS in dystonia most likely is due to a modification of the firing pattern of pallidal neurons, rather than a net reduction of their activity. ${ }^{7}$ Intraoperative microelectrode recordings in the pallidum of patients with dystonia have shown mean frequencies of $38 / \mathrm{s}$ and $46 / \mathrm{s}$ in the globus pallidus externus, and of $48 / \mathrm{s}$ and $41 / \mathrm{s}$ in the globus pallidus internus, which is lower than the firing rates in patients with Parkinson's disease. ${ }^{70}$ A higher proportion of globus pallidus internus cells in dystonia responded to somatosensory stimulation than in Parkinson's disease. ${ }^{50}$ There is limited experience on microelectrode recordings in patients with CD. Starr et al found a mean frequency of $73 \pm 12$ spikes/s in the globus pallidus internus and of $73 \pm 25$ spikes/s in the globus pallidus externus in a patient with $\mathrm{CD}^{51} \mathrm{~A}$ high proportion of discharges occurred in bursts. Similar firing patterns were seen in the present study, although we have not yet formally analyzed the microelectrode data. Cortical deactivations in dystonic patients after pallidal surgery have been shown in PET studies. ${ }^{52}$ It remains unclear why the full range of clinical improvement in dystonia occurs only after many weeks, but symptoms may recur within hours after the cessation of stimulation.

There are several problems in pallidal DBS for complex CD with current technology. Postoperative adjustment of stimulation variables can be time consuming. In particular, there may be a narrow therapeutic window for the intensity of stimulation. Our patients required multiple postoperative visits for stimulation programming. Over several months, the threshold for evoking unwanted effects increased, which allowed a gradual increase of the intensity. The amplitudes used in this study were higher than those used for treatment of patients with Parkinson's disease and patients with other movement disorders. This has also been the experience of Parkin et al, who used a mean amplitude of $4 \mathrm{~V}$ in their three patients with CD. Relatively rapid depletion of the implantable pulse generator batteries may result, especially when the Itrel II is used. The power consumption of the Itrel II is linear up to 3.6 $\mathrm{V}$, but then rises abruptly because a second capacitor is activated. ${ }^{53}$ This is another reason why we decided to replace the Itrel IIs, in those patients in whom the implantable pulse generators had to be exchanged, with the Kinetra when it become commercially available in 1999. With the Kinetra, power consumption is linear throughout the voltage range. We experienced three instances of fracture of leads in this series of patients with $C D$, which is certainly higher than in populations with other movement disorders. The connector of the extension wire to the DBS electrode is cylindrical and bulky. Because patients with other movement disorders often complained of discomfort when the connectors were placed behind and above the ear we put it in the subcutaneous tissue of the neck in the first patients in this study. The occurrence of lead fracture, however, led us to switch back again to placing the connector behind the ear.

The indications for chronic bilateral DBS of the globus pallidus internus in patients with $C D$ remain to be refined. The technique is useful in patients with complex CD who are poor candidates for peripheral procedures. Such patients include those with continuous phasic movements, marked dystonic head tremor or myoclonus, severe retrocollis, sagittal and lateral translations, and antecollis with involvement of deep cervical muscles. It may also become an alternative in patients with less complex forms of CD. It would have the advantage that also other manifestations of dystonia could be treated in the same patient, for example shoulder elevation. Furthermore, a single operation might produce the same or greater benefit than the serial procedures that are sometimes necessary with peripheral surgery. The costs of chronic DBS for treatment of CD are high, in particular because of shorter battery life and the younger age of the patients compared with those with other movement disorders. Therefore, modifications in the techniques of stimulation must be explored. With the currently available technology, battery life might be prolonged by cyclic stimulation or by cessation of stimulation overnight as long as this would not diminish clinical efficacy. Because improvement after adjusting the stimulation variables occurs only with some delay, however, it is not straightforward to assess these issues. Further investigation is also necessary with regard to the optimal stimulation site within the globus pallidus internus. With the contacts used for bipolar stimulation in this study, the target was somewhat more dorsal to that which has been used in patients with Parkinson's disease.

In conclusion, chronic DBS of the globus pallidus internus is a powerful tool for the treatment of complex CD and a useful adjunct in patients with cervical dyskinesias and secondary cervical myelopathy who must undergo spinal surgery. An absolute prerequisite for the performance of such operations is a thorough understanding of its risks and potential benefits on the part of the patient, the patient's family, and the treating physicians.

\section{ACKNOWLEDGEMENTS}

We gratefully acknowledge the collaboration of Dr Paul Heini, Department of Orthopedic Surgery, Inselspital, Berne, in the spinal reconstruction procedure in one of the patients described here.

\section{Authors' affiliations}

T Pohle, E Taub, C B Bärlocher, Department of Neurosurgery, Inselspital, University of Berne, Berne, Switzerland

T J Loher, S Weber, J-M Burgunder, Department of Neurology J K Krauss, Department of Neurosurgery, University Hospital, Klinikum Mannheim, Mannheim, Germany

\section{REFERENCES}

1 Jankovic J. Re-emergence of surgery for dystonia. J Neurol Neurosurg Psychiatry 1998;65:434.

2 Bertrand CM, Lenz FA. Surgical treatment of dystonias. In: Tsui JKC, Calne DB, eds. Handbook of dystonia. New York: Marcel Dekker, 1995:329-45.

3 Lozano AM, Kumar R, Gross RE, et al. Globus pallidus internus pallidotomy for generalized dystonia. Mov Disord 1997; 12:865-70.

4 Lin JJ, Lin GY, Shih C, et al. Benefit of bilateral pallidotomy in the treatment of generalized dystonia. Case report. J Neurosurg 1999;90:974-6.

5 lacono RP, Kuniyoshi SM, Schoonenberg T. Experience with stereotactics for dystonia: case examples. Adv Neurol 1998;78:221-6.

6 Ondo WO, Desaloms JM, Jankovic J, et al. Pallidotomy for dystonia. Mov Disord 1998;13:693-8.

7 Vitek JL, Zhang J, Evatt M, et al. Gpi pallidotomy for dystonia: clinical outcome and neuronal activity. Adv Neurol 1998;78:211-9.

8 Angelini L, Nardocci N, Estienne M, et al. Life-threatening dystonia-dyskinesias in a child: successful treatment with bilateral pallida stimulation. Mov Disord 2000;15:1010-2.

9 Coubes P, Roubertie A, Vayssiere N, et al. Treatment of DYT 1-generalised dystonia by stimulation of the internal globus pallidus. Lancet 2000;355:2220-1

10 Loher TJ, Hasdemir MG, Burgunder JM, et al. Long term follow up study of chronic globus pallidus internus stimulation for posttraumatic hemidystonia. J Neurosurg 2000;92:457-60.

11 Tronnier VM, Fogel W. Pallidal stimulation for generalized dystonia. Report of three cases. J Neurosurg 2000;92:453-6.

12 Brin MF, Jankovic J, Comella C, et al. Treatment of dystonia using botulinum toxin. In: Kurlan R, ed. Treatment of movement disorders. Philadelphia: Lippincott, 1995:183-246. 
13 Jankovic J, Brin MF. Therapeutic uses of botulinum toxin. N Engl J Med 1991;324:1186-94.

14 Jankovic J, Schwartz K. Botulinum toxin injections for cervical dystonia. Neurology 1990;40:277-80.

15 Bertrand CM. Selective peripheral denervation for spasmodic torticollis: surgical technique, results, and observations in 260 cases. Surg Neurol 1993:40:96-103.

16 Braun V, Richter HP. Selective peripheral denervation for the treatment of spasmodic torticollis. Neurosurgery 1994;35:58-62.

17 Chawda SJ, Munchau A, Johnson D, et al. Pattern of premature degenerative changes of the cervical spine in patients with spasmodic torticollis and the impact on the outcome of selective peripheral denervation. J Neurol Neurosurg Psychiatry 2000;68:465-71.

18 Ford B, Louis ED, Greene P, et al. Outcome of selective ramisectomy for botulinum toxin resistant torticollis. J Neurol Neurosurg Psychiatry 1998;65:472-8.

19 Friedman AH, Nashold BS Jr, Sharp R, et al. Treatment of spasmodic torticollis with intradural selective rhizotomies. J Neurosurg 1993;78:46-53

20 Krauss JK, Koller R, Burgunder JM. Partial myotomy/ myectomy of the trapezius muscle with an asleep-awake-asleep anesthetic technique for treatment of cervical dystonia. J Neurosurg 1999;91:889-91.

21 Munchau A, Palmer JD, Dressler D, et al. Prospective study of selective peripheral denervation for botulinum-toxin resistant patients with cervical dystonia. Brain 2001;124:769-83.

22 Krauss JK, Toups EG, Jankovic J, et al. Functional and symptomatic outcome of surgical treatment of cervical dystonia. J Neurol Neurosurg Psychiatry 1997;63:642-8.

23 Pollak L, Schiffer J, Klein C, et al. Neurosurgical intervention for cervical disk disease in dystonic cerebral palsy. Mov Disord 1998;13:713-7.

24 Waterston JA, Swash M, Watkins ES. Idiopathic dystonia and cervical spondylotic myelopathy. J Neurol Neurosurg Psychiatry 1989;52:1424-6.

25 Krauss JK, Pohle T. Historical review of functional stereotactic neurosurgery for treatment of cervical dystonia. Mov Disord 1998;13(suppl 2):134.

26 Krauss JK, Pohle T, Weber S, et al. Bilateral stimulation of globus pallidus internus for treatment of cervical dystonia [letter]. Lancet 1999:354:837-8.

27 Consky ES, Lang AE. Clinical assessments of patients with cervical dystonia. In: Jankovic J, Hallett $M$, eds. Therapy with botulinum toxin. New York: Marcel Dekker, 1994:211-37.

28 Burke RE, Fahn S, Marsden CD, et al. Validity and reliability of a rating scale for the primary torsion dystonias. Neurology 1985;35:73-7.

29 Krauss JK, Grossman RG. Operative techniques for pallidal surgery. In: Krauss JK, Grossman RG, Jankovic J, eds. Pallidal surgery for the treatment of Parkinson's disease and movement disorders. Philadelphia: Lippincott-Raven, 1998:121-33.

30 Krauss JK, King DE, Grossman RG. Alignment correction algorithm for transformation of stereotactic atlas coordinates into frame coordinates in image-guided functional neurosurgery. Neurosurgery 1998;42:806-12.

31 Taub E. Mathematical theory of stereotactic coordinate transformation: elimination of rotational targeting error by addition of a third reference point. J Neurosurg 2000;92:884-8.

32 Alkhani A, Khan F, Lang $A E$, et al. The response to pallidal surgery for dystonia is dependent on the etiology [abstract]. Neurosurgery 2000;47:504
33 Parkin S, Aziz T, Gregory R, et al. Bilateral internal globus pallidus stimulation for the treatment of spasmodic torticollis. Mov Disord 2001;16:489-93.

34 Islekel S, Zilelei $M$, Cakmur R, et al. Pallidal stimulation: an effective method in the treatment of spasmodic torticollis [abstract]. Mov Disord 2000;15(suppl 3):162.

35 Kulisevsky J, Lleo A, Gironell A, et al. Bilateral pallidal stimulation for cervical dystonia: dissociated pain and motor improvement. Neurology 2000;55:1754-5.

36 Hassler R, Dieckmann G. Stereotactic treatment of different kinds of spasmodic torticollis. Confinia Neurologica 1970;32:135-43.

37 Hassler R, Dieckmann G. Die stereotaktische Behandlung des Torticollis aufgrund tierexperimenteller Erfahrungen über die richtungsbestimmten Bewegungen. Nervenarzt 1970;41:473-8.

38 Cooper IS. Effects of thalamic lesions upon torticollis. N Engl J Med 1964;270:967-72.

39 Mundinger F. New stereotactic treatment of spasmodic torticollis with a brain stimulation system. Med Klin 1977;72:1982-6. (In German.)

40 Benabid AL, Pollak P, Gao D, et al. Chronic electrical stimulation of the ventralis intermedius nucleus of the thalamus as a treatment of movement disorders. J Neurosurg 1996;84:203-14.

41 Siegfried J, Lippitz B. Bilateral chronic electrostimulation of ventroposterolateral pallidum: a new therapeutic approach for alleviating all parkinsonian symptoms. Neurosurgery 1994;35:1126-30.

42 Villemure JG, Vingerhoets $F$, Temperli $P$, et al. Dystonia: pallidal or thalamic target [abstract]. Acta Neurochir (Wien) 2000;142:1 194.

43 Vitek JL, Giroux M. Physiology of hypokinetic and hyperkinetic movement disorders: model for dyskinesia. Ann Neurol 2000;47(suppl 1):S131-40.

44 Marsden CD, Obeso JA, Zarranz JJ, et al. The anatomical basis of symptomatic dystonia. Brain 1985;108:463-83.

45 Magyar-Lehmann S, Antonini A, Roelcke U, et al. Cerebral glucose metabolism in patients with spasmodic torticollis. Mov Disord 1997; 12:704-8

46 Naumann M, Pirker W, Reiners K, et al. Imaging of the pre- and postsynaptic side of striatal dopaminergic synapses in idiopathic cervical dystonia: a SPECT study using ${ }^{123}$ | epidepride and ${ }^{123}$ | beta-CIT. Mov Disord 1998;13:319-23.

47 Kavaklis O, Shima F, Kato M, et al. Ipsilateral pallidal control on the sternocleidomastoid muscle in cats: relationship to the side of thalamotomy for torticollis. Neurosurgery 1992;30:724-30.

48 Thompson ML, Thickbroom GW, Mastaglia FL. Corticomotor representation of the sternocleidomastoid muscle. Brain 1997:120:245-55.

49 DeToledo JC, Dow R. Sternomastoid function during hemispheric suppression by amytal: insights into the inputs to the spinal accessory nerve nucleus. Mov Disord 1998;13:809-12

50 Lenz FA, Suarez Jl, Metman LV, et al. Pallidal activity during dystonia: somatosensory reorganisation and changes with severity. J Neurol Neurosurg Psychiatry 1998;65:767-70.

51 Starr P, Christine N, Lindsey N, et al. Neuronal activity in the globus pallidus in three patients with dystonia [abstract]. Mov Disord 2000;15(suppl 3):P758

52 Kumar R, Dagher A, Hutchison WD, et al. Globus pallidus deep brain stimulation for generalized dystonia: clinical and PET investigation. Neurology 1999;53:871-4

53 Volkmann J, Fogel F, Krack P. Postoperatives neurologisches Management bei Stimulation des Nucleus subthalamicus. Akt Neurol 2000;27(suppl 1):S23-39 